\title{
Our expert panel highlights the most important research articles across the spectrum of topics relevant to the field of colorectal cancer
}

Expert panel: Franklin G Berger, Center for Colon Cancer Research, SC, USA; Cathy Eng, MD Anderson Cancer Center, TX, USA; Alexander Heriot, Peter MacCallum Cancer Centre, Australia; Bruce Minsky, MD Anderson Cancer Center, TX, USA

Kimura T, McKolanis JR, Dzubinski LA et al. MUC1 vaccine for individuals with advanced adenoma of the colon: a cancer immunoprevention feasibility study. Cancer Res. 6, 18-26 (2013).

Mobilization of the immune system occurs readily in colorectal cancer (CRC), and can lengthen time to disease recurrence and enhance survival. Such immunosurveillance can occur during the early phases of disease progression, including the preneoplastic period. The testing and utility of cancer vaccines has been hampered by difficulties in evaluating their immunogenicity and efficacy under conditions where the cancer may be causing suppression of the immune system. Among the tumor-specific molecules that have been identified for development of vaccines against CRC is MUC1, a glycosylated protein whose abnormal expression in tumors, including in the premalignant stage, drives malignancy. A number of MUC1 vaccines have been produced and examined in the therapeutic setting, and found to be well-tolerated, though only mildly immunogenic. In a recent issue of Cancer Research, Kimura et al. studied the immunogenic potential of a MUC1 vaccine in 39 patients harboring an advanced adenoma, and who were therefore at risk of developing CRC. The vaccine was found to be highly immunogenic in 17 of 39 patients $(43.6 \%)$, based on measurements of the levels of circulating anti-MUC1 antibodies 12 weeks following vaccination. Among 16 responders who were given a booster vaccination at 52 weeks,
$12(75 \%)$ exhibited a repeat response; for the 21 nonresponders, only two (9.5\%) showed a mild response to the booster. No significant toxicities were noted among the vaccinated patients. Interestingly, the research team observed significantly higher levels of circulating myeloid-derived suppressor cells in the nonresponders, a phenomenon that may have led to attenuation of the immune response, contributing to the observed absence of anti-MUC1 antibody production in these patients. The study by Kimura et al. demonstrates that vaccines directed at tumor-associated antigens such as MUC1 are immunogenic and safe in the prophylactic setting, and may be effective as preventive tools against CRC. Future efforts will focus on whether or not the anti-MUC1 vaccine is effective in inhibiting adenoma recurrence and CRC development in at-risk patients.

- Written by Franklin G Berger

Palles C, Cazier JB, Howarth KM et al. Germline mutations affecting the proofreading domains of POLE and POLD1 predispose to colorectal adenomas and carcinomas. Nat. Genet. 45, 136-144 (2013).

Germline mutations in nearly a dozen genes have been identified as contributing to familial forms of colorectal cancer (CRC). Among these, five (MSH2, MLH1, MSH6, PMS2 and MUTYH) play important roles in DNA repair. Thus, inheritance of mutant alleles in one or more of these genes puts individuals at risk for the development of
News \& Views

News

Journal Watch

Interview

Conference Scene 
CRC as a consequence of inefficient DNA repair and a propensity to accumulate mutations that can promote neoplasia. It has been observed that many individuals who appear to be predisposed to multiple or large colorectal adenomas or early-onset CRC have no detectable germline mutations in any of the known cancer-associated genes. This indicates that there may be additional genes that contribute to hereditable forms of CRC. Focusing on families with CRC, but having no mutations in known predisposing genes, Palles et al. used genome sequencing, along with linkage and association analysis, to identify variants in two new genes contributing to familial CRC. The team discovered that a Leu $\rightarrow$ Val substitution at residue 424 of POLE and a Ser $\rightarrow$ Asn substitution at residue 478 of POLD1 are associated with susceptibility to multipleadenoma and/or CRC. Both variants are highly penetrant, and neither was observed in the genomes of control, nondiseased individuals. POLE and POLD are the catalytic subunits of DNA polymerases $\varepsilon$ and $\delta$, respectively, responsible for replicative DNA synthesis. The newly identified mutations map to the so-called 'exonuclease domain' of the respective proteins, which is required for proofreading function and maintenance of replication fidelity. The amino acid substitutions likely cause deficiencies in the ability to rectify mispaired bases inserted during the replication process, thereby allowing the accumulation of mutations leading to tumor development and progression. Consistent with this was the demonstration that tumors from POLE and POLD mutation carriers exhibited high degrees of base substitution, indicative of a so-called 'mutator' phenotype. In all, the work of Palles et al. broadens the number of genes associated with familial CRC, and underscores the importance of DNA repair capacity and replication fidelity in predisposition to the disease.

- Written by Franklin G Berger

de Gramont A, Van Cutsem E, Schmoll $\mathrm{HJ}$ et al. Bevacizumab plus oxaliplatinbased chemotherapy as adjuvant treatment for colon cancer (AVANT): a Phase 3 randomised controlled trial. Lancet Oncol. 13(12), 1225-1233 (2012).

Bevacizumab is currently approved in the treatment of metastatic colorectal cancer. The role of bevacizumab was explored in the adjuvant treatment of high-risk stage II and stage III colon cancer in two large Phase III trials: AVANT and NSABP C-08 [1]. The primary end point of both trials was disease-free survival.

AVANT was a randomized threearm trial of FOLFOX $\times 12$ cycles versus FOLFOX/bevacizumab $(5 \mathrm{mg} / \mathrm{kg}) \times 12$ cycles versus xeloda $\left(1000 \mathrm{mg} / \mathrm{m}^{2}\right.$ twice a day, days $1-14) /$ oxaliplatin $\left(130 \mathrm{mg} / \mathrm{m}^{2}\right.$, day 1 ; XELOX)/bevacizumab $(7.5 \mathrm{mg} / \mathrm{kg}) \times 12$ cycles. Bevacizumab was continued for an additional 24 weeks $(7.5 \mathrm{mg} / \mathrm{kg})$ for eight cycles. In the total intention-to-treat population ( $n=3451), 2867$ patients had stage III disease, of whom 955 were randomly assigned to receive FOLFOX4, 960 to receive bevacizumab/FOLFOX4, and 952 to receive bevacizumab/XELOX. After a median of 48 months, the diseasefree survival hazard ratio for bevacizumab/ FOLFOX4 versus FOLFOX4 was 1.17 (95\% CI: 0.98-1.39; $\mathrm{p}=0.07$ ), and for bevacizumab/XELOX versus FOLFOX4 was 1.07 (95\% CI: 0.90-1.28; p = 0.44).

In a similar fashion to AVANT, the NSABP C-08 trial randomized 2672 patients with both stage II and stage III colon cancer to one of two arms: mFOLFOX6 every 2 weeks for 26 weeks or FOLFOX6 (26 weeks) + bevacizumab $(5 \mathrm{mg} / \mathrm{kg}$ every 2 weeks for 52 weeks) [1]. After a median follow-up of 35.6 months, the addition of bevacizumab to mFOLFOX6 did not result in an overall significant increase in disease-free survival (hazard ratio: 0.89; 95\% CI: 0.76-1.04; $\mathrm{p}=0.15)$.

Both AVANT and NSABP C-08 failed to identify a benefit for bevacizumab in improving the disease-free survival of early-stage colon cancer despite being given for a prolonged period after completion of 6 months of adjuvant oxaliplatin-based therapy. Therefore, the use of bevacizumab cannot be advocated in the adjuvant setting at this time. Given the lack of new developments in early-stage colon cancer therapy, other novel agents and our current approach to these early stage patients should continue to be explored.

- Written by Cathy Eng

\section{Reference \\ 1 Allegra CJ, Yothers G, O'Connell MJ et al. Phase III trial assessing bevacizumab in stages II and III carcinoma of the colon: results of NSABP protocol C-08. J. Clin. Oncol. 29(1), 11-16 (2011).}

Schoen RE, Pinsky PF, Weissfeld JL et al. Colorectal cancer incidence and mortality with screening flexible sigmoidoscopy. N. Engl. J. Med. 366, 2345-2357 (2012).

Screening for colorectal cancer has become a topic of significant debate and discussion over the last decade. The evidence for targeted population screening of individuals using fecal occult blood testing has to the implementation of national screening programs in the UK and Australia. The American College of Gastroenterologists on the other hand, recommend colonoscopy every 10 years for average-risk individuals over the age of 50 years. The benefits of endoscopic screening for colorectal cancer however, remain uncertain, although there have recently been a number of studies of the role of flexible sigmoidoscopy.

Schoen et al. report the outcome of a large randomized controlled study evaluating the effect of flexible sigmoidoscopy screening for colorectal cancer. The study was coordinated under the PLCO Cancer Screening Trial project, a multicenter study undertaken across a number of US centers. Between 1993 and 2001, 154,900 individuals between 55 and 74 years were randomized to screening with flexible sigmoidoscopy, with a repeat at between 3 and 5 years, or to usual care. Compliance with screening was high with $83.5 \%$ of 77,445 patients undergoing flexible sigmoidoscopy for primary screening and 54\% repeat screening. There was a $21 \%$ reduction in the incidence of colorectal cancer between the screening and usual care group (11.9 vs 15.2 cases per 10,000 person years respectively; $\mathrm{p}<0.001$ ) 
with significant reductions in both distal cancers (479 vs 669 cases) and proximal cancers (512 vs 595 cases). Mortality from distal colorectal cancer was significantly reduced by $50 \%$ ( 87 deaths in the screening group vs 175 deaths in the usual care group; $\mathrm{p}<0.001$ ). There was no difference in mortality from proximal colorectal cancer (143 vs 147 deaths respectively).

The findings of the study echo the findings of two previous large randomized multicentre screening trials in the UK [1] and Italy [2]. The addition of a second surveillance sigmoidoscopy in the PLCO trial increased the diagnostic yield of cancer or advanced adenoma by $26 \%$ in women and $34 \%$ in men, but it is not possible to determine the change in colorectal cancer incidence or mortality. All the trials showed that flexible sigmoidoscopy screening reduces mortality from distal colorectal cancer by reducing the cancer incidence and identifying tumors at an earlier stage. There was no evidence of a reduction in mortality from right-sided colonic tumors. This would necessitate colonoscopic screening, even though patients with abnormal findings on flexible sigmoidoscopy would undergo colonoscopy. The question is whether it is preferable to initiate a screening program with flexible sigmoidoscopy or fecal occult blood testing. The paper does address this in the discussion comparing the results of their study with the Minnesota trial of fecal occult blood testing [3], which reported a lower reduction in colorectal cancer incidence but a higher reduction in mortality with fecal occult blood testing. Direct comparison is difficult however, as it depends on the specific population, patient compliance and available resources. This will remain an area of debate.

- Written by Alexander Heriot

\section{References}

1 Atkin WS, Edwards R, Kralj-Hans I et al. Once-only flexible sigmoidoscopy screening in prevention of colorectal cancer: a multicentre randomised controlled trial. Lancet 375, 1624-1633 (2010).

2 Segnan N, Armaroli P, Bonelli L et al. Once-only sigmoidoscopy in colorectal cancer screening: follow-up findings of the Italian randomized controlled trial - SCORE. J. Natl Cancer Inst. 103, 1310-1322 (2011).

3 Mandel JS, Bond JH, Church TR et al. Reducing mortality from colorectal cancer by screening for fecal occult blood. N. Engl. J. Med. 328, 1365-1371 (1993).

Smith JD, Ruby JA, Goodman KA et al. Nonoperative management of rectal cancer with complete clinical response after neoadjuvant therapy. Ann. Surg. 256, 965-972 (2012).

Although the conventional adjuvant treatment for rectal cancer is preoperative chemoradiation there are clinical settings where surgery has not been performed. These include patients with early-stage tumors, those with medically inoperable disease and patients who have refused surgery following a favorable response to preoperative chemoradiation. Treatment of rectal cancer without surgery is not a new concept. Although patients can be cured, the results are inferior to surgery. A number of modern retrospective series report the use of radiation alone or chemoradiation, most commonly for patients who are medically inoperable or refuse surgery.

There are four modern series that advocate the watch-and-wait approach following preoperative chemoradiation [1-4]. The most recent is from Memorial Sloan Kettering and was published by Smith et al. [4]. They identified patients treated from 2006-2010 with uT2-4 and/or $\mathrm{N}^{+}$rectal cancers who received 50.4 (45-56 Gy) plus 5-fluorouracil or capecitabine. A total of 32 patients were identified who did not undergo radical surgery based on achieving a clinical complete response (defined as a negative endoscopy) at 4-10 weeks. Biopsy and imaging were optional. Of the 32 patients, seven refused surgery, seven were medically inoperable and one had concurrent lung cancer. The remainder were eligible for surgery however their physicians recommended observation. The median follow-up was 28 months (9-70 months). Adjuvant chemotherapy was given to 17 patients (53\%). The local failure rate was $19 \%$ crude and $21 \% 2$-year actuarial. The median time to local failure was 11 months and all were salvaged and without evidence of disease at 17 months. Of note, five of the six local failures were endoluminal. The incidence of distant failure was $9 \%$.

Their current recommendation for follow-up following chemoradiation is a two-stage process. At 6-7 weeks patients undergo an examination and endoscopy. If they have a clinical complete response (CR) they are followed. If they are below clinical CR then it is repeated at $10-12$ weeks. Patients who have a clinical CR at 14 months will likely remain in clinical CR.

In summary, surgery remains a standard component of the treatment of rectal cancer. The preliminary data from Smith et al. are encouraging but remain investigational. More experience, a longer follow-up, and more accurate methods for postchemoradiation assessment are needed.

- Written by Bruce Minsky

\section{References}

1 Habr-Gama A, Perez RO, Nadalin W et al. Operative vs. nonoperative treatment for stage 0 distal rectal cancer following chemoradiation therapy. Long term results. Ann. Surg. 240, 711-718 (2004).

2 Maas M, Beets-Tan RG, Lambregts DM et al. Wait-and-see policy for clinical complete responders after chemoradiation for rectal cancer. J. Clin. Oncol. 29, 4633-4640 (2011).

3 Dalton RS, Velineni R, Osborne ME et al. A single centre experience of chemoradiotherapy for rectal cancer: is there potential for nonoperative management? Colorectal Dis. 14, 567-571 (2011)

4 Smith JD, Ruby JA, Goodman KA et al. Nonoperative management of rectal cancer with complete clinical response after neoadjuvant therapy. Ann. Surg. 256, 965-972 (2012).

Financial \& competing interests disclosure

The authors have no relevant affiliations or financial involvement with any organization or entity with a financial interest in or financial conflict with the subject matter or materials discussed in the manuscript. This includes employment, consultancies, honoraria, stock ownership or options, expert testimony, grants or patents received or pending, or royalties.

No writing assistance was utilized in the production of this manuscript. 